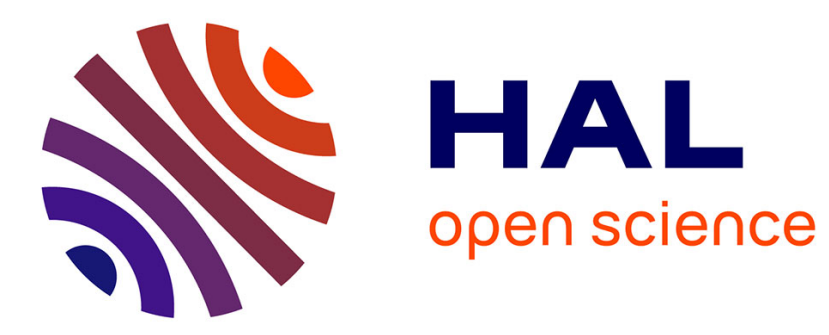

\title{
Numerical simulations of the compaction of assemblies of rubberlike particles: A quantitative comparison with experiments
}

Thi-Lo Vu, Jonathan Barés, Serge Mora, Saeid Nezamabadi

\section{- To cite this version:}

Thi-Lo Vu, Jonathan Barés, Serge Mora, Saeid Nezamabadi. Numerical simulations of the compaction of assemblies of rubberlike particles: A quantitative comparison with experiments. Physical Review E , 2019, 99 (6), 10.1103/PhysRevE.99.062903 . hal-02153495

\section{HAL Id: hal-02153495 \\ https://hal.science/hal-02153495}

Submitted on 12 Jun 2019

HAL is a multi-disciplinary open access archive for the deposit and dissemination of scientific research documents, whether they are published or not. The documents may come from teaching and research institutions in France or abroad, or from public or private research centers.
L'archive ouverte pluridisciplinaire HAL, est destinée au dépôt et à la diffusion de documents scientifiques de niveau recherche, publiés ou non, émanant des établissements d'enseignement et de recherche français ou étrangers, des laboratoires publics ou privés. 


\title{
Numerical simulations of the compaction of assemblies of rubberlike particles: A quantitative comparison with experiments
}

\author{
Thi-Lo Vu, Jonathan Barés, Serge Mora, ${ }^{*}$ and Saeid Nezamabadi \\ Laboratoire de Mécanique et de Génie Civil, UMR 5508, Université de Montpellier and CNRS, 163 Rue Auguste Broussonnet, F-34090 \\ Montpellier, France
}

(Received 20 March 2019; published 4 June 2019)

\begin{abstract}
Using the contact dymanics method together with the finite element method, we simulate the uniaxial compression of assemblies of elastic cylinders. The numerical model accounts for finite deformations of the particles through the neo-Hookean constitutive equation and solid friction between the particles. A quantitative comparison with experiments carried out with centimetric rubberlike cylinders, with local deformations of the particles determined by image correlation, is proposed. We show that the simulations accurately capture the details of both the microstructure and the macroscopic behavior of the real granular system, demonstrating the relevancy of the numerical approach.
\end{abstract}

DOI: 10.1103/PhysRevE.99.062903

\section{INTRODUCTION}

Particulate materials, composed of disordered particles, are ubiquitous in everyday life and many industrial processes, such as pharmaceuticals, foods, nuclear fuels, personal products, and cosmetics. Among these materials, some are composed of soft particles, i.e., particles that can undergo large deformations. This class includes colloidal pastes, vesicles, biological tissues, many powders, microgels, and suspensions. Many examples of such materials can be found more specifically in pharmaceutical and food industries [1,2]. Contrary to the materials composed of hard particles for which the behavior depends essentially on the particle rearrangements, the mechanical properties of soft particle materials are also intrinsically related to the local deformations of the particles. For example, the packing fraction can exceed the random close packing (RCP) $[3,4]$.

The rheological properties of soft particle systems above the RCP state remain poorly explored due to the lack of proper numerical and experimental tools. Most experimental work in this field concerns the macroscopic behaviours without access to the local mechanisms [5,6]. Numerical simulations were also performed based on the discrete element methods (DEM) with Hertz interactions (and rigid particles), but by considering arbitrary overlaps between particles (much larger than allowed by the Hertz model). Although, in these studies the global properties were quite appropriately estimated, the particle-scale parameters were not well predicted $[7,8]$. Recently some numerical works using methodologies combining features of the DEM with appropriate methods for the simulation of particle deformations were carried out [9-12].

In this paper, we investigate the compaction behavior of a granular material composed of particles able to undergo large elastic deformations. Numerical simulations combining the contact dynamics (CD) method and the finite element method

\footnotetext{
*serge.mora@umontpellier.fr
}

(FEM) are carried out and compared with experiments. This paper is organized as follows.

The system used in the experiments that will be used as a benchmark, as well as the experimental procedure, are rapidly described in Sec. II. This system consists of a bidisperse assembly of aligned cylinders of centimetric size made of a soft elastomer. They are placed together vertically at the surface of a horizontal flatbed scanner and compressed step by step in one direction by a moving vertical wall. The lower faces of the cylinders are captured by the scanner, and an image correlation procedure provides the displacement field.

The model used in the simulations is introduced in Sec. III. The simulations are carried out in plane strain conditions. This approximation for the benchmarked experimental system relies on an effective Poisson's ratio in the neo-Hookean model used to describe the mechanical behavior of the cylinders. The deformations of the cylinders are computed by means of the finite element technique, and the CD method is used for treating the contact between the particles.

Sections IV and V deal with properties defined at the scale of the whole system. After a comparison of the packing fraction as a function of the compressive strain (Sec. IV), we consider the strain energy stored in the whole system (Sec. V). The method used to measure the strain energy from the experiments is detailed. This energy is compared with the strain energy computed from the simulations in the whole range of packing fractions tested in experiments, from $78 \%$ to $88 \%$.

Sections VI and VII are devoted to quantities defined at the scale of the particles: the connectivity (Sec. VI) and the distribution of the strain energy among the particles (Sec. VII).

\section{EXPERIMENTAL METHODS}

Uniaxial compressions of bidisperse assemblies of elastic rubberlike particles were carried out with the experimental setup introduced in the previous works $[13,14]$ for studying the compression of a single particle. It consists of a compression device placed on a horizontal flatbed 


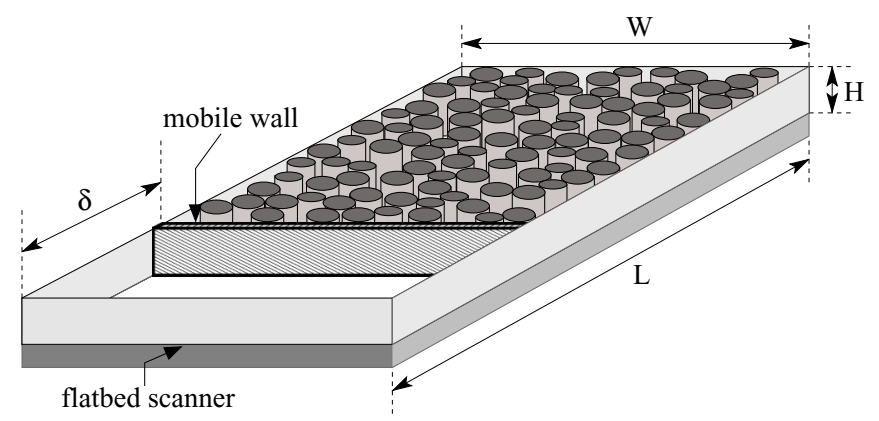

FIG. 1. Sketch of the experimental setup: a bidisperse assembly of cylindrical particles (diameters $D_{1}=20 \mathrm{~mm}$ and $D_{2}=30 \mathrm{~mm}$, height $H$ ) made of silicone rubber lays on a flatbed scanner. The system is compressed step by step by a mobile wall. The lower face of the cylinders is captured by the scanner. Dimensions of the setup are $L=286 \mathrm{~mm}, W=202 \mathrm{~mm}$, and $H=15 \mathrm{~mm}$.

scanner as shown in Fig. 1. Because of the size of the setup (length $L=286 \mathrm{~mm}$, width $W=202 \mathrm{~mm}$ ), a limited number of particles are considered (100 particles). The particles are made by curing silicone rubber in molds. Their shape is cylindrical, with two different radii, $D_{1}=20 \mathrm{~mm}$ (70 particles) and $D_{2}=30 \mathrm{~mm}$ ( 30 particles), and the same thickness $H=15 \mathrm{~mm}$. Oil, which does not swell the elastomer, is added between the particles, between the particles and the walls, and between the particles and the glass surface of the scanner. It allows lubricating the contacts and improves the optical transmission. The contact between the particles and the horizontal glass being fully lubricated, viscous-frictional contacts are obtained. However, as the oil is drained downward along the vertical surface of the cylinders, the frictional contact between the particles and between the particles and the walls has both viscous and dry components.

The cylindrical particles are bounded by four rigid vertical plates, forming a rectangle; see Fig. 1. One of the plates moves inwards with the velocity $v_{h}=2 \mathrm{~mm} \mathrm{~min}^{-1}$ by steps of $0.5 \mathrm{~mm}$, imposing on the assembly an uniaxial compression. The mobile plate is immobilized after each compressive step, and the force applied to this plate is monitored by a force sensor. For each step, once the targeted position of the mobile plate is obtained, this force decreases progressively with the time towards a constant value that is reached when the viscous forces due to lubrication are fully released. In the experiments reported here, a waiting time of $15 \mathrm{~min}$ has been fixed after each compressive step, which is enough for the relaxation of the viscous forces. Note that the measurement of the force sensor includes residual solid friction at the mobile plate; hence the confining force applied to the granular system is measured, for each step, up to an undetermined shift. The lower surfaces of the particles are scanned at the end of each rest period with a resolution of $2400 \mathrm{dpi} \times 2400 \mathrm{dpi}$ for 8-bit depth. These surfaces are coated with a shiny thin glitter layer (glitter mean size of $20 \mu \mathrm{m}$ ), consisting of a random and homogeneous pattern on the bottom of the particles with a high black-and-white contrast. This pattern is used to get the displacement field of each particle by means of a digital image correlation (DIC) algorithm introduced in previous works $[13,14]$ in which the deformations of a unique particle were investigated. In order to apply this algorithm to the case of an assembly of particles, the positions of the particles are first tracked step by step, and the rigid body motion of each particle is determined and withdrawn before the application of the DIC algorithm. Then the internal displacement field at the lower face of each cylinder is computed for every step in the compression of the system, so that the local deformation field can be determined [15].

\section{NUMERICAL MODEL}

\section{A. Constitutive equation for the particles}

The constitutive law of the silicone rubber used for the particles is known to follow the isotropic neo-Hookean model $[14,16,17]$, whose strain energy density function is

$$
\Psi=\frac{\mu}{2}\left(I_{1}-3\right)-\mu \ln J+\frac{\lambda}{2}(\ln J)^{2},
$$

with $I_{1}=\operatorname{Tr}\left(\mathbf{F}^{T} \mathbf{F}\right)$ and $J=\operatorname{det}(\mathbf{F})$. $\mathbf{F}$ is the deformation gradient tensor defined as $\mathbf{F}=\mathbf{I}+\nabla \mathbf{u}$ (I $\mathbf{I}$ being the second-order identity tensor and $\mathbf{u}$ the displacement field). $\lambda$ and $\mu$ are the Lamé parameters, and $\mu$ denotes the shear modulus. The Poisson's ratio $v$ is related to $\lambda$ and $\mu$ via $v=\lambda / 2(\lambda+\mu)$.

The shear modulus of the rubber silicone used in these experiments is $\mu=0.15 \mathrm{MPa}$ [14]. In addition, this material is almost incompressible, $v \simeq 0.5$, leading to $\lambda \rightarrow \infty$ and $J=1$ in Eq. (1). This equation therefore reduces to

$$
\Psi=\frac{\mu}{2}\left(I_{1}-3\right) \text {. }
$$

The density of the particles is $\rho=1180 \mathrm{~kg} \mathrm{~m}^{-3}$.

\section{B. Plane strain approximation}

In order to carry out numerical simulations with reasonable computation time, we consider an approximation consisting of approaching the real (experimental) systems by systems with plane-strain deformations. This amounts to reducing the $3 \mathrm{D}$ problem to a $2 \mathrm{D}$ one with a plane strain condition.

For this purpose, in order to model the experimental system made of incompressible neo-Hookean cylinders, we consider another system composed of the same particles, but (1) with effective Lamé parameters $\tilde{\lambda}$ and $\tilde{\mu}$, and (2) subjected to plane strain deformations. Let $(O x y)$ be the base plane of the system, and $(O z)$ be the out-of-plane direction. The axis of the cylinders is initially aligned along $(O z) . \tilde{\lambda}$ and $\tilde{\mu}$ should be defined so that, for the experimental system and the one with plane strain condition, their strain energy densities are equivalent, at least for infinitesimal strains. In addition, the variation of the strain energy density in the $z$ direction of the experimental system is assumed to be negligible. This assumption is based on our previous study dealing with the compression of a single rubberlike cylindrical particle [14].

Let us consider a material point located in the plane $(O x y)$, and let $\lambda_{1}=1+\varepsilon_{1}$ and $\lambda_{2}=1+\varepsilon_{2}$ be the principal stretches. The right Cauchy-Green strain tensor, $\mathbf{C}=\mathbf{F}^{T} \mathbf{F}$, is expressed in the principal coordinates system:

$$
\mathbf{C}=\left[\begin{array}{ccc}
\lambda_{1}^{2} & 0 & 0 \\
0 & \lambda_{2}^{2} & 0 \\
0 & 0 & 1 /\left(\lambda_{1}^{2} \lambda_{2}^{2}\right)
\end{array}\right] .
$$


The tensor $\mathbf{C}$ satisfies the incompressibility condition of the material: $J^{2}=\operatorname{det}(\mathbf{C})=1$ [17]. $\varepsilon_{1}$ and $\varepsilon_{2}$ have small values for infinitesimal deformations. This incompressible material being described by the neo-Hookean solid model (2), its strain energy density function is

$$
\Psi=\frac{\mu}{2}\left(I_{1}-3\right)=\frac{\mu}{2}\left(\lambda_{1}^{2}+\lambda_{2}^{2}+\frac{1}{\lambda_{1}^{2} \lambda_{2}^{2}}-3\right) .
$$

Developing this expression to order 2 in $\varepsilon_{1}$ and $\varepsilon_{2}$ gives

$$
\Psi \approx 2 \mu\left(\varepsilon_{1}^{2}+\varepsilon_{2}^{2}+\varepsilon_{1} \varepsilon_{2}\right) .
$$

Let us now consider a plane strain deformation with the right Cauchy-Green strain tensor $\tilde{\mathbf{C}}$ :

$$
\tilde{\mathbf{C}}=\left[\begin{array}{ccc}
\lambda_{1}^{2} & 0 & 0 \\
0 & \lambda_{2}^{2} & 0 \\
0 & 0 & 1
\end{array}\right]
$$

This strain tensor is no longer isochoric $[\tilde{J}=\operatorname{det}(\tilde{\mathbf{C}}) \neq 1]$. The associated strain energy density, for a compressible neoHookean material (1) with $\tilde{\lambda}$ and $\tilde{\mu}$ as Lamé parameters, is written as

$$
\tilde{\Psi}=\frac{\tilde{\mu}}{2}\left[\tilde{I}_{1}-3-2 \ln (\tilde{J})\right]+\frac{\tilde{\lambda}}{2} \ln (\tilde{J})^{2} .
$$

By developing the above expression to order 2 in $\varepsilon_{1}$ and $\varepsilon_{2}$, one obtains

$$
\tilde{\Psi} \approx\left(\tilde{\mu}+\frac{1}{2} \tilde{\lambda}\right)\left(\varepsilon_{1}^{2}+\varepsilon_{2}^{2}\right)+\tilde{\lambda} \varepsilon_{1} \varepsilon_{2} .
$$

We choose $\tilde{\mu}$ and $\tilde{\lambda}$ so that $\tilde{\Psi}=\Psi$ for any $\varepsilon_{1}$ and $\varepsilon_{2}$, which implies $\tilde{\mu}=\mu$ and $\tilde{\lambda}=2 \mu$. The effective Poisson's ratio can be then determined as

$$
\tilde{v}=\frac{\tilde{\lambda}}{2(\tilde{\lambda}+\tilde{\mu})}=\frac{2 \tilde{\mu}}{2 \times 3 \tilde{\mu}}=\frac{1}{3} .
$$

It is important to note that this approximation has been established by assuming that the deformations are infinitely small $\left(\varepsilon_{1} \ll 1\right.$ and $\left.\varepsilon_{2} \ll 1\right)$, while in the experiments the particles undergo finite deformations. In order to probe the relevancy of this 2D approach for finite deformations, numerical simulations of the radial compression of a unique cylindrical incompressible neo-Hookean particle are carried out. The results of the 3D simulation as performed in Ref. [14] are compared to ones of the 2D simulation (plane strain condition) considering the same shear modulus as $3 \mathrm{D}$, but with a Poisson's ratio of $1 / 3$.

The normalized contact force $f / D \ell \mu$ ( $D$ being particle diameter and $\ell$ its height) as a function of the compressive strain $\varepsilon$ is presented in Fig. 2. Figure 3 shows the normalized displacement field $u_{x} / D$ as a function of the Lagrangian transverse position $w$ indicated in the inset for several values of $\varepsilon$. The good agreement between these global and local results is an indication that our plane strain approximation is relevant even in the case of the finite strain.

In consequence, here the $2 \mathrm{D}$ numerical simulations in plane strain conditions were performed by considering the assemblies of compressible disklike particles with $\mu=$ $0.15 \mathrm{MPa}$ and $v=1 / 3$ (then $\lambda=0.3 \mathrm{MPa}$ ). This allows reducing considerably the computational cost.

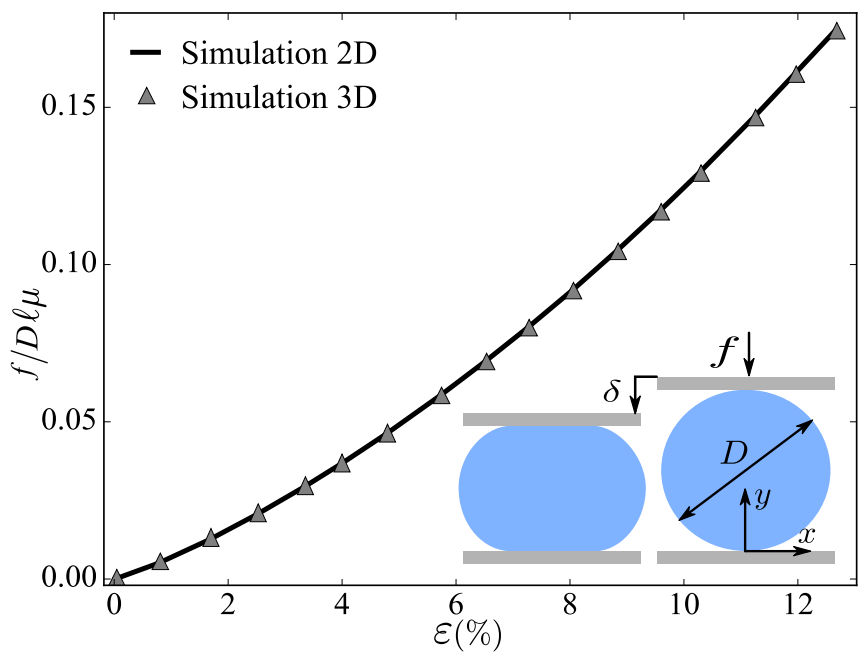

FIG. 2. Normalized contact force $f / D \ell \mu$ as a function of the compressive strain $\varepsilon[\varepsilon=-\ln (1-\delta / D)]$ for one single particle from simulations. Solid lines show the 3D numerical results with $v=0.495$ (almost incompressible neo-Hookean material), whereas the triangles present 2D simulations with $v=1 / 3$.

\section{Numerical implementation}

The numerical simulations were performed using a nonlinear finite element model implemented in the LMGC90 code [18]. This model is based on coupling an implicit FEM formulation and the CD method. In the FEM-CD procedure, each particle is discretized by finite elements for accounting for particle deformations, and the frictional contact interactions between particles are treated by means of the CD method.

As mentioned before, to carry out the simulations in a reasonable computation time, 2D simulations in plane strain conditions are considered. The numerical assemblies are defined by considering the same initial geometry (position, radius, and number of particles). The particles are meshed using the GMSH software [19]. The relative spatial resolution for each particle is $\kappa=0.071\left(\kappa=\frac{d}{D_{i} / 2}\right.$, where $d$ is the average size of the elements and $D_{i}$ the particle diameter). Two particle sizes

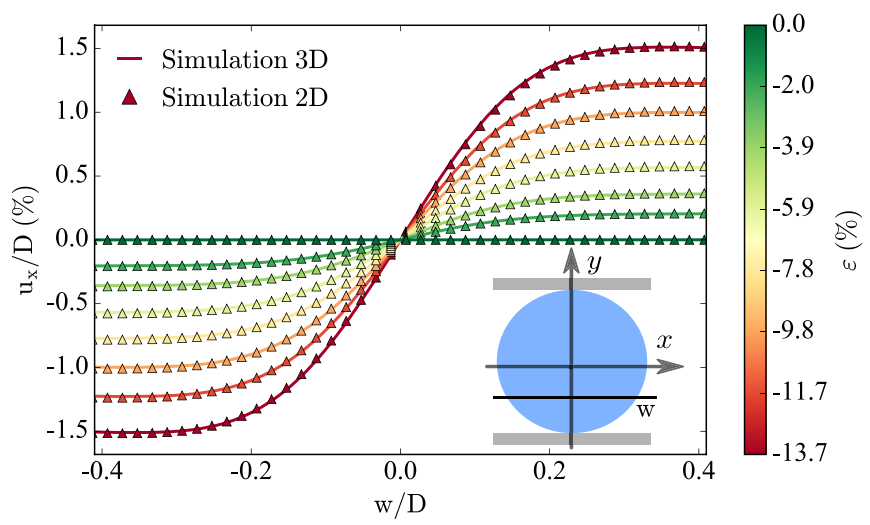

FIG. 3. Dimensionless displacement field along the $x$ direction $u_{x} / D$ as a function of the Lagrangian transverse position $w$ shown in inset, for several values of $\varepsilon$. Solid lines show the $3 \mathrm{D}$ numerical results with $v=0.495$ (almost incompressible neo-Hookean material), whereas the triangles show the $2 \mathrm{D}$ numerical results with $v=1 / 3$. 
are considered for the simulations as the experiments. The small particles, $D_{1}=20 \mathrm{~mm}$, are meshed with 670 three-node triangular elements and the large ones, $D_{2}=30 \mathrm{~mm}$, with 1506 three-node triangular elements. The walls are modeled as undeformable solid objects.

Since the experimental system is analyzed at the end of each compression step after a long relaxing time following the piston immobilization, because of energy dissipation (due to viscoelasticity inside the particles and the viscous friction between the particles and the scanner surface), all the velocities are then zero. In the simulations, a Rayleigh damper [20] is hence added to the model in order to reproduce an overdamped regime. The Rayleigh damping matrix is a linear combination of the mass matrix $\mathbf{M}$ and the stiffness matrix $\mathbf{K}$ :

$$
\mathbf{C}_{\text {Rayleigh }}=\alpha \mathbf{M}+\beta \mathbf{K},
$$

where $\alpha$ and $\beta$ are the relative damping coefficients of mass and stiffness of the system, respectively. The mass proportional damping effect is dominant for low frequencies and the stiffness proportional damping for high frequencies.

In order to avoid abrupt changes in the velocities in the simulations, the system is subjected to an uniaxial compaction by moving the boundary corresponding to the mobile plate, at a constant velocity $v_{h}$. The quasistatic condition is approached (1) by fixing the constant velocity $v_{h}$ much slower than the elastic waves celerity $(\sim \sqrt{\mu / \rho})$ and (2) by choosing damping coefficients in the Rayleigh damper so that the elastic waves and velocities are overdamped and do not have significant effects on the results. A piston velocity of $0.1 \mathrm{~m} \mathrm{~s}^{-1}, \alpha=$ $30 \mathrm{~s}^{-1}$, and $\beta=1 \times 10^{-5} \mathrm{~s}$ fulfill these requirements. The time step in the simulations is $\delta t=9 \mu \mathrm{s}$.

Experiments and numerical simulations of uniaxial compression of bidisperse assemblies composed of the same cylindrical particles with the same initial configuration have been repeated for three equivalent assemblies, but with different initial positions of particles. In the following, quantities will be averaged over these three initial states in order to obtain less noisy information and a more general behavior of this granular media.

Measuring experimentally a priori the coefficient of friction $\mu_{f}$ (between particles and between particles and walls) is a difficult task because it depends on the amount and on the distribution of lubricant oil among the particles. Since the same amount of oil is added under the same conditions to the three different experimental assemblies, the coefficient of friction $\mu_{f}$ is not expected to change for the three different configurations. In the following, numerical simulations are performed with different coefficients of friction in order to retain the one that mimics better the experimental results.

Simulation of the compression of the granular system, from an initial compacity of $78 \%$ to a final compacity of $88 \%$ in 355 compression steps requires a computational time of about $120 \mathrm{~h}$ with a $2.4 \mathrm{GHz}$ processor.

\section{PACKING FRACTION AND CUMULATIVE COMPRESSIVE STRAIN}

The packing fraction $\Phi$ is defined here as the ratio between the total area of the particles projected on the horizontal plane and the total horizontal area of the system. In the experiments,

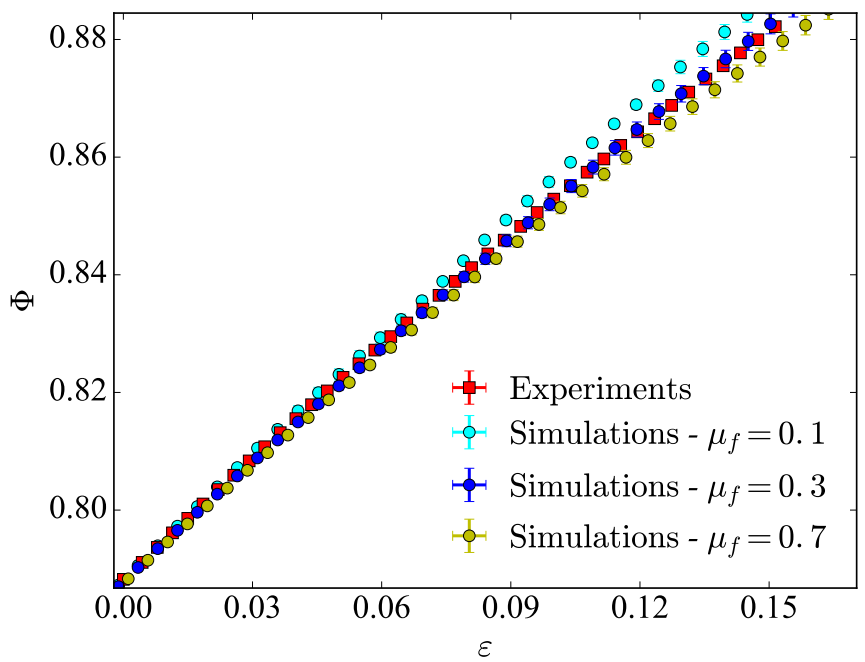

FIG. 4. Packing fraction $\Phi$ as a function of the cumulative compressive strain $\varepsilon$ for the experiments, and the numerical results for the coefficients of friction $\mu_{f}=0.1, \mu_{f}=0.3$, and $\mu_{f}=0.7$.

$\Phi$ is directly measured from the raw images obtained from the scanner. The current total area of the box and the area of the particles are measured with ImageJ software, for different values of the cumulative compressive strain $\varepsilon$, defined by

$$
\varepsilon=\left|\ln \left(\frac{\text { Final length }}{\text { Initial length }}\right)\right|=-\ln \left(1-\frac{\delta}{L}\right) .
$$

The absolute value $|\cdot|$ is to obtain a positive value of $\varepsilon$ when the system is compressed. $\varepsilon$ is deduced from the displacement $\delta$ of the piston (which is also measured from the raw images). Figure 4 shows the packing fraction $\Phi$ as a function of the cumulative compressive strain $\varepsilon$ for the numerical and experimental assemblies. In the numerical tests, the same coefficient of friction $\mu_{f}$ is assigned between the particles and between the walls and the particles. Several values of $\mu_{f}$ are considered. The simulations with $\mu_{f}=0.3$ reproduce quasiperfectly the experimental results; see Fig. 4.

\section{STRAIN ENERGY STORED IN THE SYSTEM}

A part of the energy provided to the system by the piston during the compression is stored through the elastic deformation of the particles. The remaining part is dissipated by friction. The strain energy stored in the particles for the whole system is equal to the integral of the strain energy density [see Eq. (2)] computed on the domain composed of all the particles. It is determined experimentally from the strain field obtained from the DIC, which gives the displacement field of the material point located at the lower surface of the particles. It has been demonstrated in Ref. [14] that the strain energy of incompressible neo-Hookean cylinders diametrically compressed can be computed from this 2D displacement fields for strains up to $16 \%$. The approximation consists of approaching the true deformation with an invariant deformation along the axis of the cylinder. Let $F_{2 D}(x, y)$ be the tensor defined by

$$
\mathbf{F}_{2 D}(x, y)=\left[\begin{array}{cc}
1+\frac{\partial u_{x}}{\partial x} & \frac{\partial u_{x}}{\partial y} \\
\frac{\partial u_{y}}{\partial x} & 1+\frac{\partial u_{y}}{\partial y}
\end{array}\right]
$$




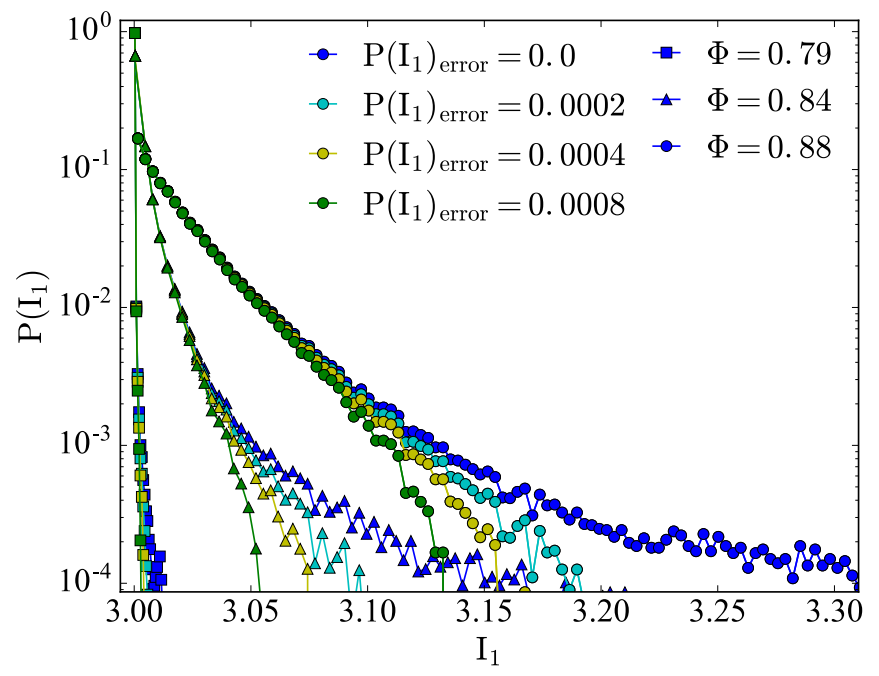

FIG. 5. Blue symbols: Probability density functions for invariant $I_{1}$ calculated from DIC for different levels of packing fraction $(\Phi=$ $[0.79,0.84,0.88])$. Symbols with other colors are obtained by subtracting a constant error value, $\mathrm{P}\left(I_{1}\right)_{\text {error }}$, to the previous probability density functions.

and let $\lambda_{x}^{2}$ and $\lambda_{y}^{2}$ be the eigenvalues of $\mathbf{C}_{2 D}=\mathbf{F}_{2 D}^{T} \mathbf{F}_{2 D} . \lambda_{x}$ and $\lambda_{y}$ are assumed to approach the in-plane eigenvalues of the right Cauchy-Green strain tensor $\mathbf{C}$. Because the material is incompressible, the product of the three eigenvalues of $\mathbf{C}$ has to be equal to unity: $J=1$. Hence, we take for the outof-plane eigenvalue $\lambda_{z}^{2}=1 /\left(\lambda_{x}^{2} \lambda_{y}^{2}\right)$. Following Ref. [14], the strain energy density $\Psi(x, y, z)$ of the incompressible material is then approximated by taking

$$
I_{1}(x, y)=\lambda_{x}^{2}+\lambda_{y}^{2}+1 /\left(\lambda_{x}^{2} \lambda_{y}^{2}\right)
$$

in Eq. (2).

Some erroneous values of the displacement calculated with the DIC procedure can happen if a particle has moved with a large rotation angle or its image has bad contrast in the final state. These inaccurate displacement values lead to dubious evaluations of $I_{1}(x, y)$ at few locations. $I_{1}(x, y)$, defined by Eq. (13), is computed for each image point (pixel) by the DIC procedure. The probability density function (PDF) of $I_{1}(x, y), \mathrm{P}\left(I_{1}\right)$, is presented in Fig. 5 for three different packing fractions of one system. By construction, all the values of $I_{1}(x, y)$ are larger than 3 , which is the value of $I_{1}$ in the absence of deformation. $\mathrm{P}\left(I_{1}\right)$ is maximum for $I_{1}=3$ and decreases as $I_{1}$ increases; see Fig. 5. This decrease is less and less pronounced as $I_{1}$ increases, leading to nonzero values of $\mathrm{P}\left(I_{1}\right)$ that are too large to be physically relevant. One therefore subtracts to the PDF a constant value, $\mathrm{P}\left(I_{1}\right)_{\text {error }}$, so that these nonphysical values are removed. Indeed, below a given value of the probability density, the PDFs are noisier. Taking this value for $\mathrm{P}\left(I_{1}\right)_{\text {error }}$, the decrease of the new PDF is more regular. From the corrected PDF defined as

$\mathrm{P}\left(I_{1}\right)_{\text {corrected }}=\left[\mathrm{P}\left(I_{1}\right)-\mathrm{P}\left(I_{1}\right)_{\text {error }}\right] / \int\left[\mathrm{P}\left(I_{1}\right)-\mathrm{P}\left(I_{1}\right)_{\text {error }}\right] d I_{1}$,

the value of the strain energy $\mathcal{E}$ of the system is obtained. The normalized strain energy, $\mathcal{E} /(W H L \mu)$ is shown in Fig. 6 (red squares) as a function of the packing fraction $\Phi$. The error bars

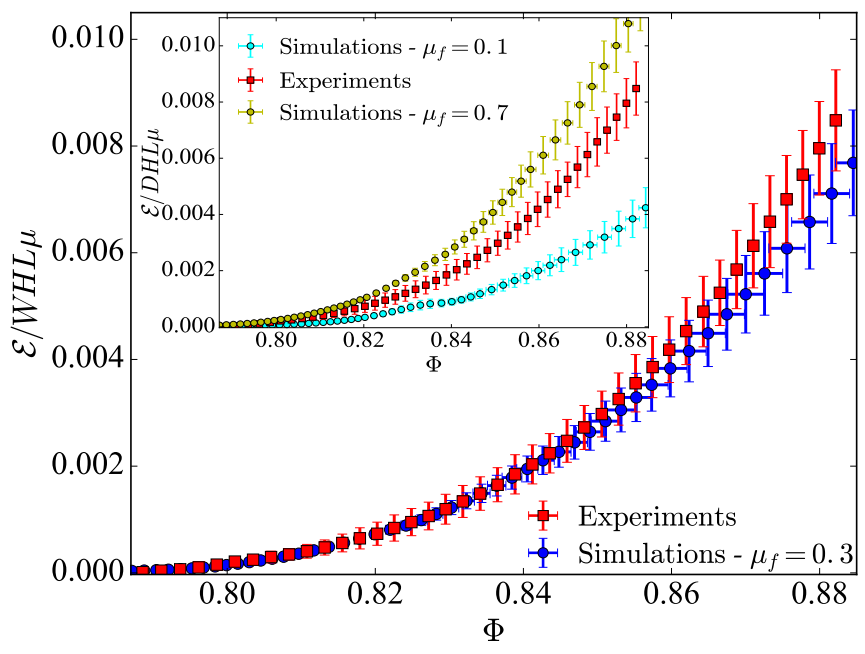

FIG. 6. Normalized total strain energy $\mathcal{E} / W L H \mu$ as a function of the packing fraction $\Phi$ from the experiments, and from the numerical simulations with the coefficient of friction $\mu_{f}=0.3$. Inset: Normalized total strain energy $\mathcal{E} / W L H \mu$ as a function of the packing fraction $\Phi$ from the experiments, and from the numerical simulations coefficients of friction $\mu_{f}=0.1$ and $\mu_{f}=0.7$.

originate both from the uncertainty in the choice of $\mathrm{P}\left(I_{1}\right)_{\text {error }}$ (between 0.0002 and 0.0007 as deduced from Fig. 5) and from the differences between the three initial configurations.

The strain energy obtained from the numerical simulations can thus be compared with the experimental result. The best accordance is found for the coefficient of friction $\mu_{f}=0.3$ (see Fig. 6), in accordance with the conclusion of Sec. IV.

We show below that this comparison can be extended to quantities defined at the particle scale, as the connectivity (Sec. VI) and the distribution of the strain energy among the particles (Sec. VII). In what follows, the coefficient of friction is fixed to be $\mu_{f}=0.3$ according to the conclusion of Secs. IV and $\mathrm{V}$.

\section{CONNECTIVITY}

Figure 7 presents three snapshots of the compaction of a neo-Hookean particle assembly obtained by numerical simulations (top figures) and the experiments (bottom figures). These two assemblies have the same initial configuration. Due to the intrinsically disordered nature of these systems and their high sensitivity to fluctuations, numerical simulations and experiments do not lead to identical states at the individual particle scale, as shown by the different positions of particles or force chains. In order to be able to compare usefully these two systems at the particle scale, we will focus on mean local quantities of the assemblies.

The connectivity $Z$ is defined as the average number of force-bearing contacts per particle. The inactive contacts and floating particles are therefore not taken into account in the connectivity. In the experiments, the force-bearing contacts are identified because of the local deformation of the particle determined by the DIC procedure. Due to experimental noise, the local deformation determined by the DIC procedure may not be rigorously equal to zero even at inactive contacts. A strain energy density threshold $\Psi^{*}$ is then chosen in order to 


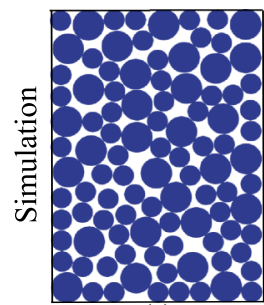

(a)

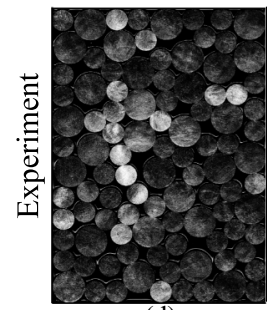

(d)

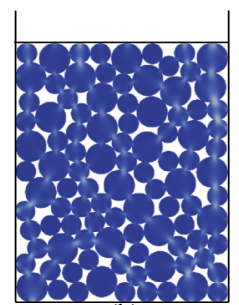

(b)

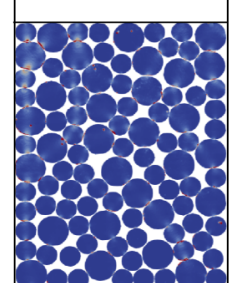

(e)

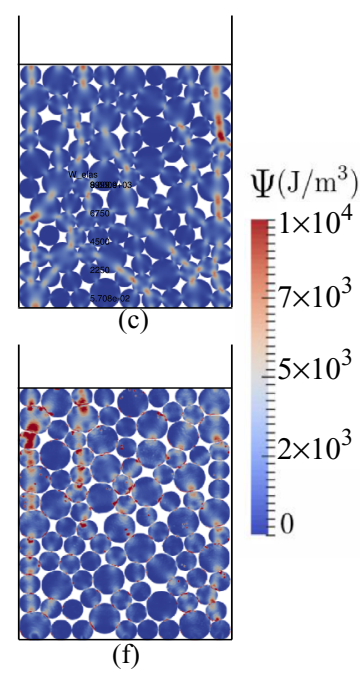

FIG. 7. Snapshots of the uniaxial compression of an assembly of elastic cylinders for the numerical simulations (a-c) with $\mu_{f}=0.3$ and the experiments $(\mathrm{d}-\mathrm{f})$. The packing fractions of the systems are $\Phi=0.78$ (a, d), $\Phi=0.83$ (b,e), $\Phi=0.88$ (c, f). Snapshot (d) shows the initial positions of particles in the experimental setup. The strain energy density $\Phi$ represented with the same color code in panels (a)(c) and (e) and (f).

discriminate whether a contact is active: if the strain energy density at the vicinity of the contact between two particles is larger than $\Psi^{*}$, the contact is considered as active. In order to test the influence of $\Psi^{*}$ on the obtained value of $Z$, the measured $Z$ is plotted in the inset of Fig. 8 as a function of $\Phi$ for $\Psi^{*}$ in the range $\left[6 \times 10^{-3} \mu ; 6 \times 10^{-5} \mu\right]$. The lowest

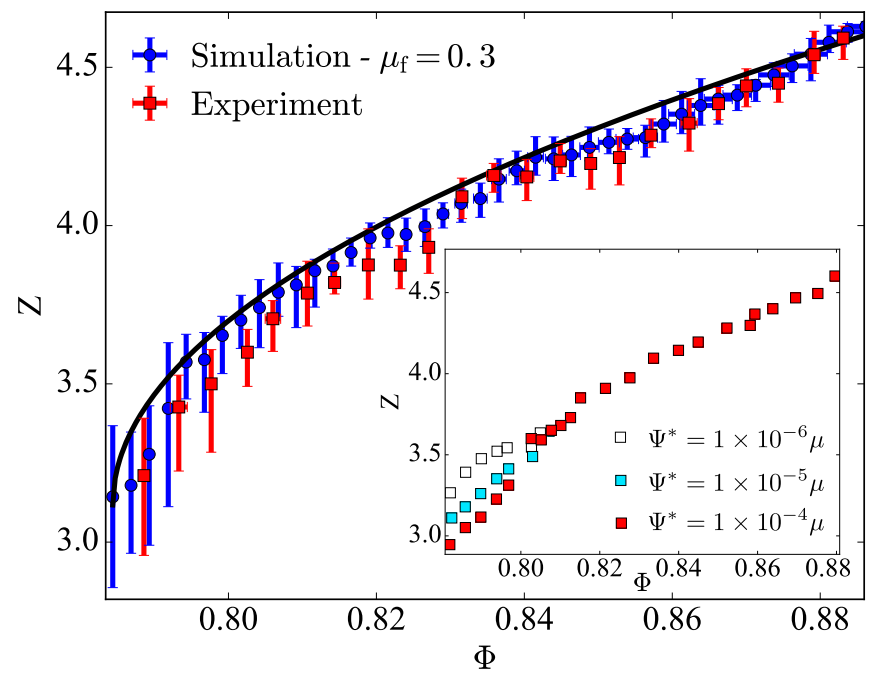

FIG. 8. Connectivity $Z$ as a function of the packing fraction $\Phi$ determined from the experiments and the numerical simulations. The solid line is the power-law fit [see Eq. (14)] with $\Phi_{T}=0.785 \pm$ $0.005, \Phi_{1}=0.9, Z_{T}=3$, and $Z_{1}=4,72$. Error bars result from the dispersion over three different initial configurations and for the experimental data, from the connectivity measurement procedure. Inset: Connectivity obtained for different energy thresholds $\left(\Psi^{*}=\left[6 \times 10^{-3} \mu, 6 \times 10^{-4} \mu, 6 \times 10^{-5} \mu\right]\right)$ for a given initial configuration. value, $\Psi^{*}=6 \times 10^{-5} \mu$, is an estimation of the experimental resolution for the strain energy density, and the largest value, $6 \times 10^{-3} \mu$, is high enough compared to the noise level to be sure to exclude any nonactive contact. In Fig. 8 the error bars arise both from the changes in the measured $Z$ upon changes of $\Psi^{*}$ in the range $\left[6 \times 10^{-3} \mu ; 6 \times 10^{-5} \mu\right]$ and from the dispersion over the three experimental configurations.

In the simulations, since a constant velocity $v_{h}$ of the piston was imposed, possible residual velocities of some particles may remain. In order to exclude contacts originating from nonstatic events (as the deformation induced by the rebound of a floating particle), the mechanical equilibrium of the particles is probed as follows: If the magnitude of the resultant contact forces $f$ applied to a given particle is larger than a threshold $f_{p}^{*}$, the particle is excluded for the determination of the connectivity. The threshold $f_{p}^{*}$ is chosen to be proportional to the total compressive force $f$ of the system. With a coefficient of proportionality ranging from $0.05 \%$ to $0.1 \%$, no significant change in the connectivity is observed. Moreover, since the contact is computed on the nodes, a contact is taken into account for the computation of $Z$ if it occurs at least in two nodes of each particle.

The numerical and experimental connectivities $Z$ as a function of the packing fraction $\Phi$ are shown in Fig. 8. We observe a quantitative agreement between the two approaches; $Z$ increases when $\Phi$ increases. This increase results from mechanisms that can be separated as follows: at lower packing fractions, particles can rearrange to find equilibrium state and, therefore, easily get new neighbors; at higher packing fractions, $Z$ increases due to elastic particle deformation.

The variation of $Z$ with the packing fraction has been studied for various systems such as emulsions, foams, and solid particle assemblies [4,21-23], leading to the phenomenological relation:

$$
Z=Z_{T}+K \sqrt{\Phi-\Phi_{T}},
$$

where $\Phi_{T}$ is the value of the packing fraction at the rigidity transition, i.e., the packing fraction corresponding to the appearance of the mechanical rigidity of the whole system. $Z_{T}$ is the connectivity for $\Phi=\Phi_{T}$. As mentioned in Ref. [11], the prefactor $K$ can be set to be

$$
K=\left(Z_{1}-Z_{T}\right) / \sqrt{\left(\Phi_{1}-\Phi_{T}\right)},
$$

where $Z_{1}$ and $\Phi_{1}$ correspond to any state after the rigidity transition. The experimental and numerical results are fitted with Eq. (14) by setting $\Phi_{1}=0.9, Z_{1}=4.72$, and $Z_{T}=3$, which corresponds to the isostaticity condition for frictional particles [4], yielding to $\Phi_{T}=0.785 \pm 0.005$. Note that this result complements the literature data for elastic particle assemblies with the packing fraction much larger than $\Phi_{T}$. Indeed, previous work reported experiments carried out on elastic particle systems over a narrow range of packing fraction [21,24] or DEM simulations based on Hertz-Mindlin approximations that cannot describe correctly the behavior of elastic particles subjected to large deformations $[4,23,25,26]$.

\section{DISTRIBUTION OF STRAIN ENERGY}

In Sec. $\mathrm{V}$ the total strain energy of the assembly was studied as a function of the packing fraction of the system. 


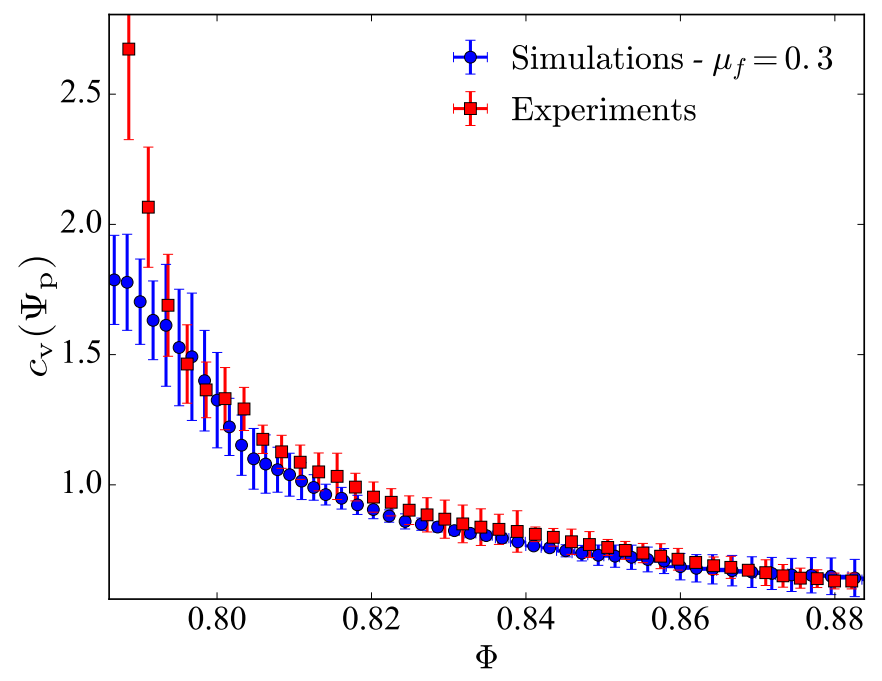

FIG. 9. Relative standard deviation of the mean particle strain energy density, $c_{v}\left(\Psi_{p}\right)$, as a function of the packing fraction $\Phi$, calculated from the experiments and from the numerical simulations. Error bars on the numerical data result from the dispersion over the three different initial configurations. Error bars on the experimental data result both from the dispersion over the three initial configurations and from the DIC energy measurement procedure (see Sec. V).

Here we consider the distribution of the mean strain energy density of the particles. The mean strain energy density $\Psi_{p}$ of a particle $p$ is strain energy of the particle divided by its initial volume. $\Psi_{p}$ is zero when particle $p$ is not deformed and increases with the deformation. $\Psi_{p}$ can vary from one particle to another. The relative standard deviation $\left(c_{v}\right)$ of $\Psi_{p}$, defined as

$$
c_{v}\left(\Psi_{p}\right)=\frac{\sqrt{\left\langle\Psi_{p}^{2}\right\rangle-\left\langle\Psi_{p}\right\rangle^{2}}}{\left\langle\Psi_{p}\right\rangle},
$$

gives a measure of the heterogeneity of the particle strain energy density. Here $\langle\cdot\rangle$ denotes the average over the particles of the system.

Figure 9 shows $c_{v}\left(\Psi_{p}\right)$ as a function of $\Phi$, obtained from experiments and numerical simulations. The same procedure as in Sec. V is applied in order to get reliable determinations of the energy density. The agreement between experiments and simulations is quantitatively good except for $\Phi<0.79$. Indeed, for packing fractions smaller than, or close to, the critical rigidity packing fraction of the system, $\Phi_{T} \simeq 0.79$, residual velocities of the particles create small, but not negligible, deformations into the particles, altering $c_{v}\left(\Psi_{p}\right)$ compared to the static case. For larger packing fractions, the small deformations that may result from a residual velocity are negligible, and the agreement with the experiments becomes satisfactory. Note that the fact that $c_{v}\left(\Psi_{p}\right)$ decreases with $\Phi$ shows that the distribution of the strain energy among particles, or equivalently the distribution of the deformations, is less and less heterogeneous as the packing fraction increases beyond the critical rigidity packing fraction.

\section{CONCLUSION}

In this paper, we have shown that the behavior of an assembly of vertical elastic cylinders undergoing a uniaxial horizontal compression of finite amplitude can be quantitatively captured by numerical simulations based on contact dynamics and the finite element method.

Starting from the incompressible neo-Hookean model to describe the finite deformations of the elastic cylinders, we have proposed an approximation relying on an effective neoHookean constitutive law with a finite compressibility and plane strain deformations.

Fixing the coefficient of friction to $\mu_{f}=0.3$ in the simulations makes it possible to reproduce the variations of the packing fraction and the total strain energy as a function of the global compressible strain. The analysis of quantities at the particle scale, as the connectivity and the strain energy distribution, also leads to a good agreement between experiments and numerical simulations.

We have demonstrated that the heterogeneities in the strain energy density are less and less pronounced as the packing fraction increases beyond the rigidity transition packing fraction, and we have shown that the connectivity can be well described, up to large packing fractions, by the relation previously established for other assemblies of deformable particles.

The numerical method can be applied to wide variety of systems, such as systems composed of particles with different initial shapes, constitutive laws, or polydispersities. In order to characterize more deeply the mechanical response of these granular systems, simulations of systems composed of higher numbers of particles would be useful, as well as other geometries, such as pure shear or biaxial compression.

\section{ACKNOWLEDGMENTS}

The authors thank M. Renouf and F. Dubois for interesting discussions. This work was partially supported by Labex NUMEV (ANR-10-LABX-20). The authors state that there are no conflicts of interest.
[1] E. Emery, J. Oliver, T. Pugsley, J. Sharma, and J. Zhou, Flowability of moist pharmaceutical powders, Powder Technol. 189, 409 (2009).

[2] M. Barnabé, N. Blanc, T. Chabin, J.-Y. Delenne, A. Duri, X. Frank, V. Hugouvieux, E. Lutton, F. Mabille, S. Nezamabadi, N. Perrot, F. Radjai, T. Ruiz, and A. Tonda, Multiscale modeling for bioresources and bioproducts, Innovative Food Sci. Emerging Technol. 46, 41 (2018).
[3] M. Cloitre, R. Borrega, F. Monti, and L. Leibler, Glassy Dynamics and Flow Properties of Soft Colloidal Pastes, Phys. Rev. Lett. 90, 068303 (2003).

[4] M. van Hecke, Jamming of soft particles: Geometry, mechanics, scaling and isostaticity, J. Phys.: Condens. Matter 22, 033101 (2010).

[5] J. Zhang, T. S. Majmudar, M. Sperl, and R. P. Behringer, Jamming for a 2D granular material, Soft Matter 6, 2982 (2010). 
[6] P. Menut, S. Seiffert, J. Sprakel, and D. A. Weitz, Does size matter? Elasticity of compressed suspensions of colloidal- and granular-scale microgels, Soft Matter 8, 156 (2012).

[7] R. T. Bonnecaze and M. Cloitre, Micromechanics of soft particle glasses, Adv. Polym. Sci. 236, 117 (2010).

[8] A. Favier de Coulomb, M. Bouzid, P. Claudin, E. Clément, and B. Andreotti, Rheology of granular flows across the transition from soft to rigid particles, Phys. Rev. Fluids 2, 102301 (2017).

[9] S. Nezamabadi, F. Radjai, J. Averseng, and J.-Y. Delenne, Implicit frictional-contact model for soft particle systems, J. Mech. Phys. Solids 83, 72 (2015).

[10] S. Nezamabadi, T. H. Nguyen, J. Y. Delenne, and F. Radjai, Modeling soft granular materials, Granular Matter 19, 8 (2017).

[11] S. Nezamabadi, X. Frank, J. Y. Delenne, J. Averseng, and F. Radjai, Parallel implicit contact algorithm for soft particle systems, Comput. Phys. Commun. 237, 17 (2018).

[12] G. Mollon, Mixtures of hard and soft grains: Micromechanical behavior at large strains, Granular Matter 20, 39 (2018).

[13] S. Mora, T.-L. Vu, J. Barés, and S. Nezamabadi, Highly deformed grain: From the Hertz contact limitation to a new strain field description in 2D, EPJ Web Conf. 140, 05011 (2017).

[14] T. L. Vu, J. Barés, S. Mora, and S. Nezamabadi, Deformation field in diametrically loaded soft cylinders, Exp. Mech. 59, 453 (2019).

[15] T.-L. Vu and J. Barés, Soft grain compression: Beyond the jamming point, arXiv:1903.09979.
[16] G. A. Holzapfel, Nonlinear Solid Mechanics: A Continuum Approach for Engineering (Wiley, London, 2000).

[17] S. Nezamabadi, H. Zahrouni, and J. Yvonnet, Solving hyperelastic material problems by asymptotic numerical method, Comput. Mech. 47, 77 (2011).

[18] LMGC90, Lmgc90 (2018), https://git-xen.lmgc.univ-montp2. fr/lmgc90/lmgc90_user/wikis/home.

[19] GMSH, Gmsh (2018), http://gmsh.info/.

[20] J. W. Strutt and B. Rayleigh, The Theory of Sound, 2nd ed. (Dover, New York, 1945).

[21] T. S. Majmudar, M. Sperl, S. Luding, and R. P. Behringer, Jamming Transition in Granular Systems, Phys. Rev. Lett. 98, 058001 (2007).

[22] D. J. Durian, Foam Mechanics at the Bubble Scale, Phys. Rev. Lett. 75, 4780 (1995).

[23] C. S. O'Hern, L. E. Silbert, A. J. Liu, and S. R. Nagel, Jamming at zero temperature and zero applied stress: The epitome of disorder, Phys. Rev. E 68, 011306 (2003).

[24] H. A. Makse, D. L. Johnson, and L. M. Schwartz, Packing of Compressible Granular Materials, Phys. Rev. Lett. 84, 4160 (2000).

[25] C. S. O'Hern, S. A. Langer, A. J. Liu, and S. R. Nagel, Random Packings of Frictionless Particles, Phys. Rev. Lett. 88, 075507 (2002).

[26] K. Shundyak, M. van Hecke, and W. van Saarloos, Force mobilization and generalized isostaticity in jammed packings of frictional grains, Phys. Rev. E 75, 010301(R) (2007). 